\title{
LOCOMOCIÓN HUMANA: MODELOS Y VARIABLES BIOMECÁNICAS
}

\section{HUMAN LOCOMOTION: MODELS AND BIOMECHANICAL VARIABLES}

\author{
Carlo Massimo Biancardi ${ }^{1}$, Renata Luisa Bona ${ }^{1}$ y Leonardo Lagos Hausheer ${ }^{1,2}$
}

carlo.biancardi@gmail.com; renatalbona@gmail.com; lagoskinesiologo@gmail.com

\author{
${ }^{1}$ Laboratorio de Investigación en Biomecánica y Análisis del Movimiento, Departamento de \\ Ciencias Biológicas, Centro Universitario Paysandú, CENUR Litoral Norte, Universidad de la \\ República de Uruguay, Paysandú, Uruguay. \\ ${ }^{2}$ Departamento de Kinesiología, Facultad de Medicina, Universidad de Concepción, Chile
}

Envío original: 2020-04-09 Reenviado: 2020-06-19, 2020-08-04 Aceptado: 2020-08-20

Publicado: 2020-09-08

Doi: https://doi.org/10.15517/pensarmov.v18i2.41360

\begin{abstract}
RESUMEN
La locomoción con miembros implica diferentes desafíos de tipo mecánico: en cada ciclo los segmentos tienen que ser reposicionados anatómicamente, con fases de aceleración y deceleración, con la consiguiente elevación y descenso del centro de masa. Dentro de la locomoción humana la marcha y la carrera son actividades fundamentales en todo el ciclo vital de las personas. En específico la carrera es una actividad muy estudiada por las ciencias del deporte, medicina, fisioterapia o kinesiología, ingeniería y biólogos, por el alto impacto articular y muscular que genera, ya que esto trae consigo lesiones y alteraciones en el rendimiento de los pacientes y deportistas. El constante estudio de la locomoción a reconocido diferentes desafíos de tipo fisiológico y mecánico tanto en la explicación de fenómenos como en el análisis de estos. Este artículo busca aclarar conceptos a través de textos clásicos, que se consideran básicos e importantes para entender las investigaciones posteriores. Además, se explican diferentes parámetros que sirven para comprender la locomoción, tanto en estudios con humanos como en animales. El objetivo de este trabajo es hacer una revisión de la literatura, que se enfoca en los aspectos mecánicos, energéticos, el efecto del tamaño y los diferentes patrones de la locomoción,
\end{abstract}


con sus modelos mecánicos. Finalmente, los autores esperan que este texto sea un aporte para docentes y estudiantes para la comprensión de la locomoción en idioma español.

Palabras clave: marcha, carrera, mecánica, biomecánica.

\begin{abstract}
Walking and running are fundamental activities throughout the life cycle of people. Specifically, running is a highly studied activity due to the high joint and muscular impact it generates in those who practice, commonly causing injuries and alterations in the performance of athletes. Locomotion generates different physiological and mechanical challenges. This article seeks to clarify concepts mentioned in classic texts, which are basic and important to understand the research that followed. It also tries to explain different parameters that help to understand locomotion, contrasting with studies in humans and animals. The objective of this work is to review the literature, which focuses on the mechanical and energetic aspects of locomotion, the effect of size and different patterns of locomotion, with their mechanical models.
\end{abstract}

Keywords: walking, running, mechanics, biomechanics.

\title{
INTRODUCCIÓN
}

Los animales terrestres se desplazan principalmente gracias al movimiento cíclico de las extremidades. Si bien existe una técnica alternativa para la locomoción en tierra, es decir el rastreo, que se basa en la contracción de la musculatura axial, la locomoción con miembros es también la forma más antigua de locomoción terrestre (Biancardi, Fabrica, Polero, Loss, y Minetti, 2011).

La locomoción con miembros implica diferentes desafíos de tipo mecánico: en cada ciclo los segmentos tienen que ser reposicionados anatómicamente, con fases de aceleración y deceleración, con la consiguiente elevación y descenso del centro de masa (Cavagna, 2017b). Este artículo busca aclarar conceptos a través de estudios clásicos, explicar diferentes parámetros que sirven para comprender la locomoción, y discutir algunos estudios desarrollados con animales. Estos son importantes para entender las investigaciones posteriores. El objetivo de este trabajo es hacer una revisión de la literatura, que discute aspectos mecánicos y energéticos de la locomoción, el efecto del tamaño y los diferentes patrones, con sus modelos mecánicos. 


\section{LOCOMOCIÓN BÍPEDA Y CUADRÚPEDA}

Los humanos desarrollamos en nuestros actos locomotores tres actividades que permiten movilizarnos, y que compartimos con los otros bípedos y los cuadrúpedos: caminar, correr (o trotar) y galopar (skipping) (Saibene y Minetti, 2003). En los humanos caminar se reconoce porque existe una fase de contacto de ambos pies en el suelo, lo mismo pasa en los pares delantero y trasero de los cuadrúpedos caminando. Correr está determinado por la ausencia del doble apoyo y la presencia de una fase balística, o de vuelo, más o menos predominante. En los cuadrúpedos el mismo movimiento, con un desfase del $50 \%$ entre los pares delantero y trasero, determina el trote. El galope o "skipping" se distingue por estar compuesto por un paso con doble contacto, seguido de un salto (fase de vuelo), y en los cuadrúpedos representa un patrón eficiente para moverse a gran velocidad. En los humanos el galope se desarrolla cuando somos niños, o cuando bajamos una colina, incluso es la forma de locomoción utilizada por los astronautas cuando existe menor aceleración de gravedad (Minetti, 1998; Pavei, Biancardi y Minetti, 2015).

Los procesos mecánicos y metabólicos son diferentes entre caminar, galopar y correr, y todos provocan diferentes costos energéticos en la locomoción (Margaria, 1976; Margaria, Cerretelli, Aghemo y Sassi, 1963). A diferencia de los cuadrúpedos donde trotar es tan económico como galopar, para los humanos realizar el galope es más costoso que correr (Hoyt, Wickler, Dutto, Catterfeld, y Johnsen, 2006; Minetti, Ardigò, Reinach y Saibene, 1999; Minetti, Pavei y Biancardi, 2012; Pavei et al., 2015).

La comparación de los costos de funcionamiento entre los humanos y otras especies animales ha sido de gran interés para los científicos para entender relaciones entre estructura y función de las extremidades (Roberts, Kram, Weyand y Taylor, 1998). Taylor, Schmidt-Nielsen y Raab (1970) estimaron que el costo energético de carrera en humanos ( $C$ run, $\mathrm{J} \mathrm{kg}^{-1} \mathrm{~m}^{-1}$, [ver la sección "Costo de transporte"]) es dos veces mayor que el valor predicho para un animal de la misma masa. Fedak y Seeherman (1979) y luego, Taylor, Heglund y Maloiy (1982) informaron que en realidad el costo sería cercano a $43 \%$ más que el predicho por factores alométricos, los cuales se definen como la relación entre las proporciones del cuerpo y el tamaño total de este (Ranisavljev, llic, Soldatovic y Stefanovic, 2014). La principal conclusión de estos estudios es que la carrera humana tiene un alto costo comparado al trote y galope de los cuadrúpedos. Lo contrario pasa con la marcha: caminar es más económico en los humanos que en los cuadrúpedos de masa similar (Bramble y Lieberman, 2004). En general se acepta que el costo de transporte en la marcha de los humanos es más bajo y el de la carrera es más alto que en los animales de masa similar (Aiello y Wells, 2002; Bramble y Lieberman, 2004; Carrier et al., 1984; Leonard y Robertson, 1997; Rodman y McHenry, 1980; Schmidt-Nielsen, 1975; Steudel- 
Numbers, 2003; Steudel, 1996; Taylor, 1994). Alexander (2003) derivó una ecuación predictiva del costo de transporte en relación a la masa corporal basada en los datos de Taylor et al. (1982). La figura 1 presenta el costo de transporte de varias especies en relación con la masa corporal y la ecuación predictiva de Alexander (2003).

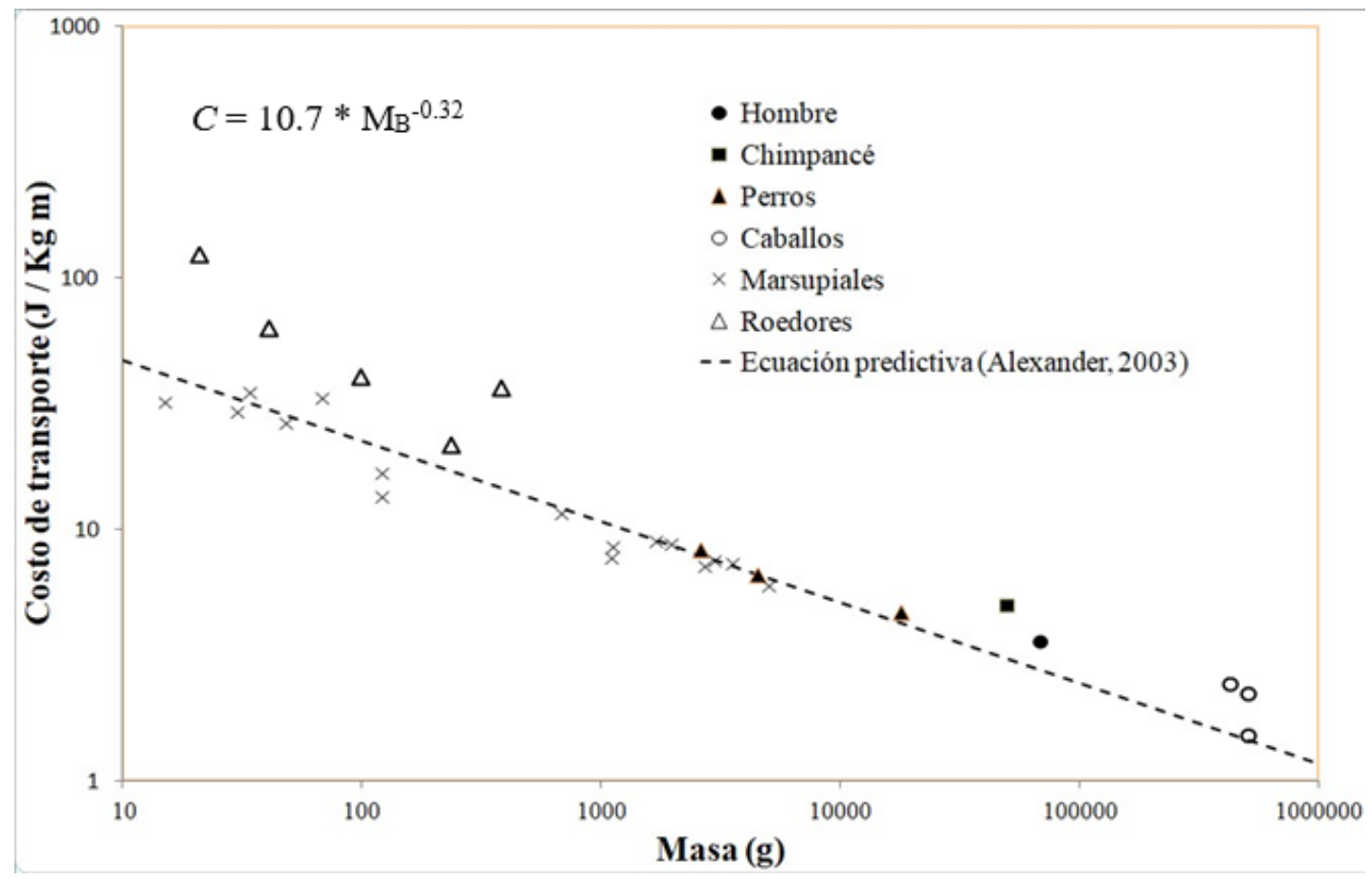

Figura 1. Costo de transporte $(C)$ en relación de la masa corporal en algunos mamíferos terrestres y la ecuación predictiva de Alexander (2003),$C=10.7{ }^{*} \mathrm{M}_{\mathrm{B}}{ }^{-0.32}$ donde $C=$ Costo de transporte $(\mathrm{J} / \mathrm{kg} / \mathrm{m}), M_{B}=$ Masa corporal $(\mathrm{kg})$. El $C$ decrece al aumentar de la masa con una pendiente alrededor de -0.32 (los ejes se presentan en escala logarítmica). Fuente: elaboración propia a partir de (Minetti et al., 1999; Pontzer, Raichlen y Sockol 2009; Taylor et al., 1970; Taylor et al., 1982; Wunder y Morrison, 1974).

Desde el punto de vista histórico, el pionero en analizar aspectos biomecánicos humanos de la carrera, y marcha, fue Margaria (1938), el cual estudió el efecto de diferentes velocidades e inclinaciones. En la década del 40 los investigadores Wallace Fenn y Herbert Elftman hicieron muchas contribuciones, tales como el estudio de los cambios en energía cinética $\left(E_{K}\right)$ y energía potencial $\left(E_{P}\right)$, y métodos para calcular la fuerza en actividades de locomoción (Elftman, 1940; Fenn, 1941). El mismo Margaria en la década del 60 analizó aspectos como el costo energético usando un nomograma o nomógrafo, un instrumento grafico de cálculo bidimensional que 
permitía calcular fácilmente el gasto de energía en función de la velocidad e inclinación (Margaria, Cerretelli, Aghemo y Sassi, 1963). Posteriormente Cavagna, Saibene y Margaria (1963; 1964) volvieron a investigar las características mecánicas en la carrera y la marcha. Cavagna y Kaneko (1977) mejoraron las estimaciones de trabajo mecánico de la carrera considerando la transferencia de energía cinética entre los segmentos para el cálculo del trabajo interno. Después Minetti, Ardigò y Saibene (1994) concluyeron que existe un aumento en el trabajo mecánico total en la carrera con inclinación. No obstante, los valores obtenidos de trabajo mecánico para la carrera sobreestiman el trabajo muscular realizado debido a la dificultad de cuantificar la energía elástica durante ese movimiento (Saibene y Minetti, 2003).

Fue en los años 70 del siglo XX que Robert McNeill Alexander empezó a utilizar la fórmula del número de Froude ( $n F r$ ) (ecuación 1) para comparar aspectos mecánicos y energéticos de la locomoción en especies diferentes o en sujetos de tamaño diferente dentro de la misma especie (Alexander, 1976)

$$
n F r=v^{2} / g L
$$

Donde $v$ es la velocidad, $g$ es la aceleración de gravedad y $L$ la longitud del miembro inferior.

El $n F r$ es un número adimensional parte de un modelo denominado similitud dinámica, que asume que dos cuerpos geométricamente semejantes, cuyos movimientos se basan en el intercambio entre $E_{K}$ y $E_{P}$, se comportan de forma semejante dinámicamente y se mueven al mismo número de Froude (Alexander y Jayes, 1983). Posteriormente Alberto Minetti aplicó el concepto de similitud dinámica en diferentes estudios de comparación de marcha en poblaciones con diferentes características (Minetti, Saibene, et al., 1994) y también comparando la marcha a diferentes valores de aceleración gravitacional (Minetti, 2001b; 2001a). La fórmula de Froude determina un límite superior a la velocidad de marcha (caminar), equivalente a $n F r=1$; se supone que la velocidad espontánea de transición marcha-carrera (o marcha-trote en los cuadrúpedos) ocurre a $n F r=0.5$; la velocidad adimensional que corresponde a la velocidad óptima de marcha es el $n F r=0.25$ (Alexander, 2003).

\section{MECÁNICA DE LOS MIEMBROS}

El esqueleto de los vertebrados funciona como un sistema de palancas articuladas, donde los músculos producen movimientos y transmiten fuerza al desarrollar momentos en las articulaciones (torque). Los músculos sólo pueden generar fuerza de tracción, entonces la flexión y la extensión de una articulación son desarrolladas por conjuntos antagónicos de músculos 
(McMahon, 1984) o por el mismo grupo muscular trabajando de manera concéntrica y excéntrica, por ejemplo, la flexión de rodilla después del contacto inicial del pie con el suelo durante la marcha o la carrera es controlada principalmente por el cuádriceps actuando de manera excéntrica. En una articulación el brazo de palanca $(r)$, que es la distancia perpendicular desde el vector de la fuerza muscular al eje de rotación de la articulación, influye sobre el torque ejercido $(M)$ por el músculo, como sobre la velocidad angular de la articulación $(\omega)$ y su rango de movimiento. Sin embargo, $M$ depende de una relación directa de $r$ con la fuerza del músculo (F) ecuación (2).

$$
M=F r
$$

Entonces ( $r$ ) varía conforme la articulación gira ya que el ángulo que forma la línea de acción de la fuerza (dirección entre el origen y la inserción) y el segmento corporal con respecto al cual se está midiendo el radio cambia al girar el segmento. Mientras que $\omega$ varía inversamente con $r$ (ecuación 3)

$$
\omega=v_{s} / r
$$

Siendo "Vs" la tasa de acortamiento de las fibras musculares. En consecuencia, el torque que un músculo puede desarrollar varía inversamente con el rango de movimiento de la articulación y la velocidad del movimiento articular que puede producir. La mejor composición de fuerza y velocidad depende del nivel de la relación fuerza-velocidad del músculo mismo (Margaria, 1976; McMahon, 1984), como de la relación fuerza-velocidad resultante del mecanismo de palanca (Alexander, 2003; Biewener y Patek, 2018; McMahon, 1984).

Cuando el pie entra en contacto con el suelo, se puede medir la fuerza de reacción (G). El componente vertical $\mathrm{G}_{\mathrm{z}}$ se opone a la aceleración de gravedad y provoca la aceleración en este eje, mientras que los componentes antero-posterior y medio-lateral ( $G x$ y Gy) representan la fricción en el plano horizontal, que permiten al hombre o animal acelerar, desacelerar, maniobrar y equilibrarse. $Y$ además son las fuerzas que permiten el avance hacia el frente o hacia los lados (ver figura 2). 
Marcha

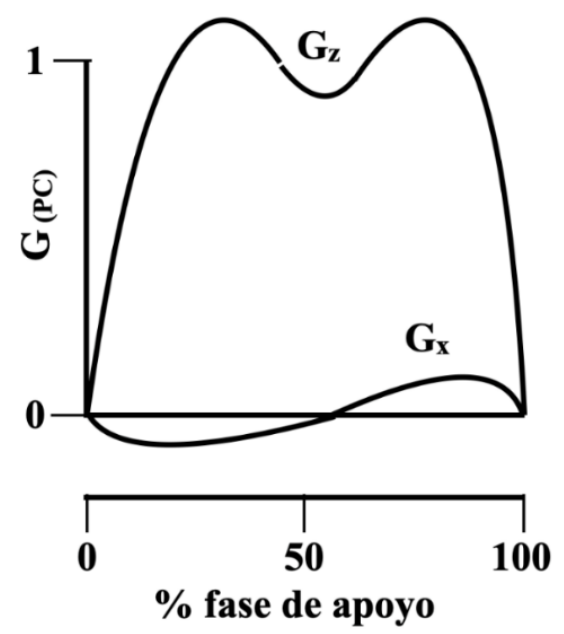

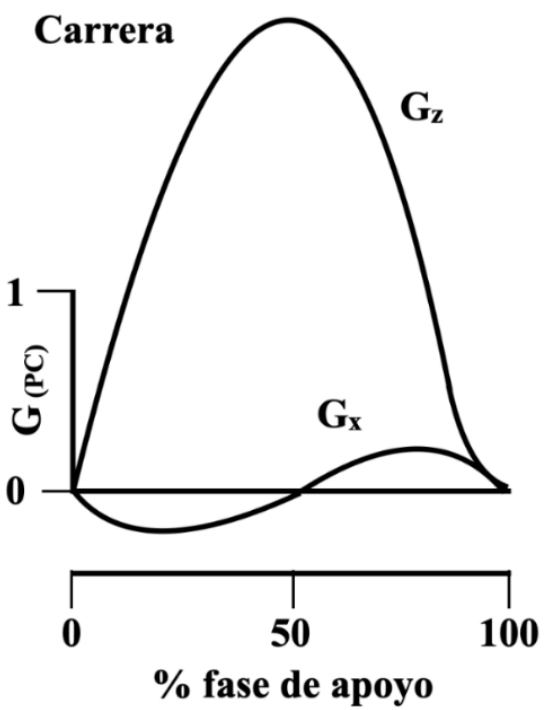

$\%$ fase de apoyo

Figura 2. Fuerzas de reacción contra el piso en un paso de marcha y de carrera. $G(P C)$ : Fuerza expresada como fracción del peso corporal; Gz: Componente vertical de G; Gx: componente antero-posterior de G; en el eje horizontal el tiempo expresado como porcentaje del tiempo de apoyo. El área bajo las curvas representa el impulso. Fuente: elaboración propia.

En reposo, la fuerza $G_{z}$ que actúa sobre cada miembro es aproximadamente igual al peso corporal dividido por el número de miembros que lo soportan. Cuando un animal se mueve, independientemente del tipo de paso y de la velocidad, las extremidades empujan al suelo acelerando el cuerpo en la dirección deseada, durante la fase llamada de apoyo. Luego, los miembros deben ser reposicionados para dar un paso adelante, dejando el suelo y acelerando también con respecto al centro de masa del cuerpo, en la fase llamada de oscilación. Durante la locomoción, las fuerzas ejercidas por una extremidad en el suelo suben y bajan durante la fase de apoyo (cuando el pie está en contacto con el suelo), y es cero cuando la extremidad está en fase de oscilación, la fuerza vertical promedio ejercida por la suma de los miembros sobre un ciclo completo zancada tiene que ser igual al peso corporal para mantenerlo a una altura relativa del suelo. Se deduce que, si los miembros se mantienen en el suelo durante un período de tiempo más corto, se ejercerán fuerzas mayores sobre cada miembro (Alexander, 2003; Biewener, 1989).

La fracción relativa de una zancada cuando una extremidad particular está en la fase de apoyo se define como el Duty Factor de la extremidad $\left(D_{F}\right)$ (Alexander, $\underline{1989 \mathrm{~b}}$ ). Para moverse más rápido, un animal tiene que mover sus miembros más rápidamente, aumentando la 
frecuencia de la zancada, y/o tiene que tomar pasos más largos, aumentando la longitud de la zancada, acoplando una o más fases de vuelo, cuando no hay miembros en fase de apoyo. En cualquier caso, el aumento de la velocidad de locomoción se logra reduciendo el $D_{F}$ y aumentando así la magnitud de la fuerza ejercida contra el suelo.

Debido a que los momentos que actúan en una articulación se tienen que equilibrar en condiciones estáticas o a velocidad angular constante, siendo $F$ fuerza muscular, $G$ fuerza de reacción, $r$ el brazo de palanca y $R$ la carga de la resistencia (ecuación 4):

$$
F r=G R
$$

Reordenando la ecuación (4):

$$
r / R=G / F
$$

Estas proporciones de brazos de palanca y fuerzas proporcionan una medida de la Ventaja Mecánica Efectiva (VME) de los músculos de las extremidades (figura 2). La postura afecta la VME y entones la alineación de los huesos articulados en la dirección de la fuerza de reacción del suelo cambia la ventaja mecánica, reduciendo la fuerza muscular empleada (Biewener, 1989; 1990), esto sucede cuando R disminuye. Tales cambios en la VME de las articulaciones de los miembros son importantes para permitir que mamíferos de diferentes tamaños mantengan el pico de tensión muscular y ósea dentro de un intervalo estrecho y, por tanto, tengan un factor de seguridad (Safety Factor) similar. El factor de seguridad a menudo se define en términos de la resistencia de una estructura en relación con la tensión máxima probable que experimente durante su vida útil. El estrés óseo y muscular depende del área transversal de los huesos y de las fibras musculares, que varían proporcionalmente a la masa corporal (Alexander, Jayes, Maloiy, y Wathuta, 1981). Por lo tanto, la reducción relativa a la masa en el pico de las fuerzas musculoesqueléticas se logra, en animales de gran tamaño, mediante la adopción de una postura más vertical, lo que cambia la VME (ver figura 3) (Biewener, 1989). 

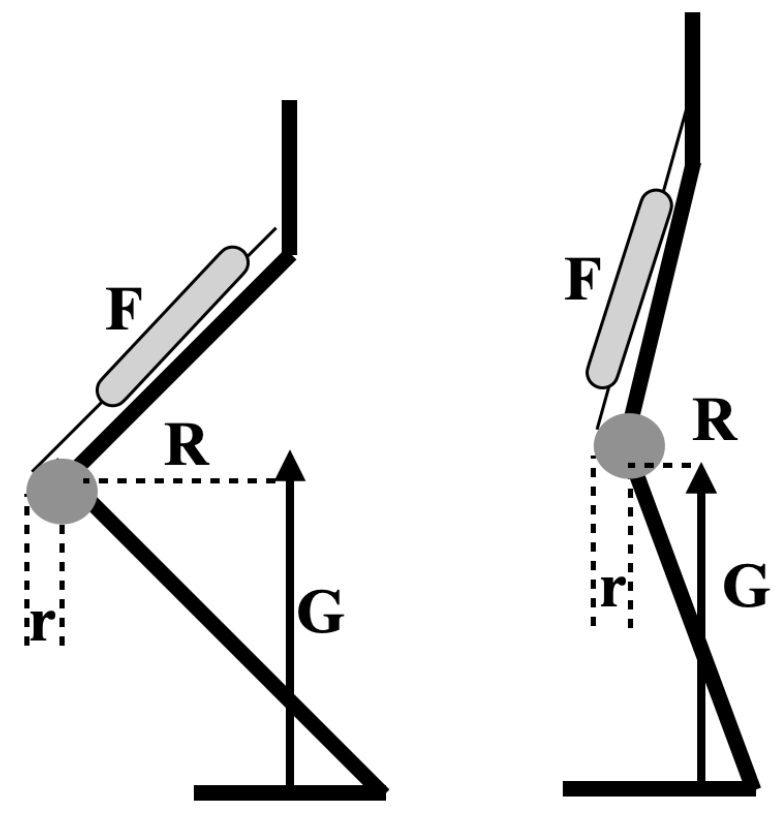

Figura 3. Esquematización de un miembro inferior humano. G: Fuerza de reacción del suelo (ground reaction force); R: brazo de la fuerza G; F: fuerza del musculo cuádriceps; r: brazo de la fuerza F. La VME (r/R) a la rodilla resulta mayor en la postura más erguida (a la derecha). Fuente: elaboración propia.

\section{TRABAJO MECÁNICO}

Al final entonces, los músculos convierten energía química producida por el metabolismo (energía metabólica, $E_{M e t}$ ) en trabajo mecánico $(W)$. En biomecánica de la locomoción se considera " $W_{\text {TOT" }}$ " el trabajo mecánico total hecho para mover los segmentos corporales, y desplazar todo el cuerpo. No se consideran los movimientos a nivel de tejido (fibras) muscular. Por supuesto hay casos donde los músculos convierten $E_{M e t}$ sin generar $W_{T O T}$, por ejemplo, en caso de contracciones isométricas o co-contracciones de músculos agonistas y antagonistas para estabilizar una articulación. Sin embargo, está generalmente aceptado que, durante la locomoción, el gasto energético $E_{\text {Met }}$ sea proporcional al trabajo mecánico $W_{\text {TOT }}$ (Cavagna, 2017b).

De acuerdo con Cavagna (2017a), ver la ecuación 5:

$$
W_{T O T}=W_{E X T}+W_{I N T}
$$


Donde $W_{E X T}$ es el trabajo externo, hecho para levantar y desplazar el centro de masa de todo el cuerpo ( $\mathrm{b}_{\mathrm{COM}}$ ), y $W_{\text {INT }}$ es el trabajo interno, hecho para mover y reposicionar en cada ciclo los miembros.

El cálculo del trabajo mecánico se basa en el teorema trabajo-energía, el cuál afirma que el trabajo mecánico hecho por un sistema en un dado proceso resulta ser la diferencia entre la energía mecánica total a final del proceso y la energía mecánica total (EMT) al inicio del proceso (ecuación 6):

$$
W=E M T_{F}-E M T_{1}
$$

Siendo la $E M T=E_{P}($ Energía potencial gravitacional $)+E_{K}($ Energía cinética $) . E M T_{F}=$ energía mecánica total final; $E M T_{l}=$ energía mecánica total inicial.

Si se suman los aumentos de EMT del $b_{C o M}$ en el tiempo se determina el trabajo externo positivo ( $W_{E X T}$ ) (Cavagna y Kaneko, 1977; Cavagna et al., 1963; Willems, Cavagna y Heglund, 1995). El trabajo necesario para rotar y acelerar las extremidades respecto al $b_{c o m}$ corresponde al $W_{\text {INT. }}$. El $W_{\text {INT }}$ constituye 25 a $40 \%$ del $W_{\text {TOT }}$ (Cavagna, 2017a; Willems et al., 1995).

Dividiendo el $W_{\text {TOT }}$ por la $E_{\text {Met }}$ se obtiene la eficiencia mecánica de la locomoción (Eff). Dado que, como se explica en la siguiente sección, el gasto metabólico de la locomoción generalmente se expresa como costo de transporte $\left(C ; \mathrm{J} \mathrm{kg}^{-1} \mathrm{~m}^{-1}\right)$, por coherencia también el trabajo mecánico se divide por la masa del sujeto y por la distancia recorrida (Minetti et al., 1999).

\section{COSTO DE TRANSPORTE}

El Costo de Transporte $(C)$, es el costo metabólico necesario para mover una unidad de masa para una unidad de distancia (Schmidt-Nielsen, 1975). Fue estudiado en la marcha humana por Margaria en 1963 (Margaria et al., 1963). El C (ecuación 7) se estima con medidas de calorimetría indirecta, es decir, con medidas de consumo de oxígeno y/o producción de dióxido de carbono. Generalmente se prefiere convertir los mililitros de oxígeno a Joules porque es la unidad de medida de la energía en el sistema internacional, esto se realiza a través de un factor de conversión que depende del cociente respiratorio $\left(\mathrm{mlCO}_{2} / \mathrm{mlO}_{2}\right)$ (Di Prampero, 2015).

$$
C\left(\frac{J}{\text { kg.m }}\right)=\frac{\left(\operatorname{Vo2total}\left(\frac{m l .02}{\text { kg.s }}\right)-\operatorname{Vo2reposo}\left(\frac{m l .02}{k g s}\right)\right)}{\text { velocidad }\left(\frac{m}{s}\right)} \cdot \operatorname{Eq}(R)\left(\frac{J}{m l .02}\right)
$$


Donde $C$ es el costo de transporte en $\mathrm{J} \mathrm{kg}^{-1} \mathrm{~m}^{-1}, \mathrm{VO}_{2}$ total $\left(\mathrm{ml} \mathrm{O}_{2} \mathrm{~kg}^{-1} \mathrm{~s}^{-1}\right)$ es el consumo de oxígeno obtenido con un ergoespirómetro, $\mathrm{VO}_{2}$ reposo $\left(\mathrm{ml} \mathrm{O}_{2} \mathrm{~kg}^{-1} \mathrm{~s}^{-1}\right)$ es el consumo de oxígeno obtenido en reposo en bípedo durante 5 minutos, Velocidad $\left(\mathrm{m} \mathrm{s}^{-1}\right)$ es la velocidad media alcanzada y $\mathrm{Eq}(\mathrm{R})\left(\mathrm{J} \mathrm{ml}^{-1} \mathrm{O}_{2}^{-1}\right)$ es el factor de conversión.

Se considera que, en sujetos adultos sin cambios del patrón de marcha, el mínimo $C$ es cercano a los $2 \mathrm{~J} \mathrm{~kg}^{-1} \mathrm{~m}^{-1}$, y coincide con una velocidad de marcha óptima, muy cerca de la velocidad determinada por el ritmo de caminata natural (autoseleccionada) (Saibene y Minetti, 2003). El C aumenta cuando el valor de la velocidad es menor o mayor que el valor óptimo, determinando una característica curva con forma de "U", cuando se presenta el C en función de la velocidad (ver figura 4).

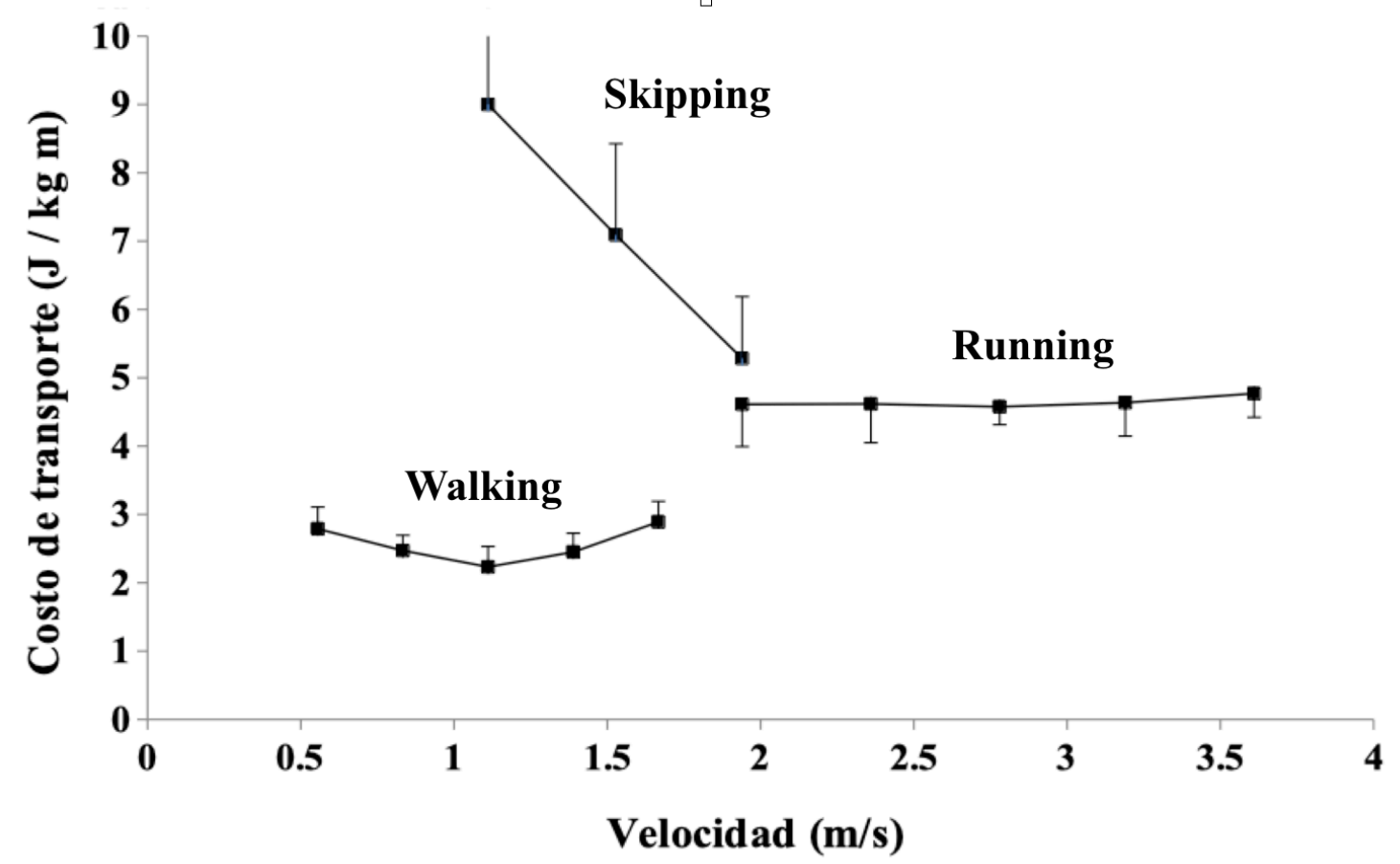

Figura 4. Costo de transporte $(C)$ en relación del patrón y de la velocidad en el hombre. Fuente: elaboración propia basado en (Pequera et al., 2020).

En los adultos mayores la curva del costo se desplaza hacia arriba, es decir que el $C$ aumenta con la velocidad (Mian, Thom, Ardigò, Narici y Minetti, 2006). La velocidad autoseleccionada u óptima depende del tamaño según la fórmula de Froude (ecuación 1), y coincide con un $\mathrm{nFr}=0,25$ (Saibene y Minetti, 2003). Algunas condiciones o enfermedades pueden resultar en un alto $C$ con alteraciones de su curva de distribución, con divergencias entre las velocidades de mínimo $C$ y velocidad autoseleccionada (Bona et al., 2017; 2016; Bona, 
Gomeñuka, Storniolo, Bonezi, y Biancardi, 2019; Detrembleur, Dierick, Stoquart, Chantraine, y Lejeune, 2003; Detrembleur, Vanmarsenille, De Cuyper, y Dierick, 2005). Estas condiciones cambian en ambientes de poca o nula aceleración de gravedad, como por ejemplo en la luna, donde se observa una reducción del trabajo mecánico y por lo tanto una disminución del $C$. Sin embargo, también la velocidad óptima y la velocidad límite de marcha bajan con la aceleración de gravedad, de acuerdo con la fórmula de Froude (ecuación 1), determinando por ejemplo la adopción de un patrón de galope en vez de marcha durante el desplazamiento de los astronautas en la luna (Minetti et al., 2012; Pavei et al., 2015). Consideración importante puesto que en la actualidad existen terapias incipientes de rehabilitación de marcha basadas en condiciones de peso reducido o aceleración gravitacional reducida (Alkner y Bring, 2019; Salisbury, Baptista, Fei, Susin y Russomano, 2015).

Como se ha afirmado antes, en condiciones terrestres el galope tiene un $C$ muy alto, comparado con la marcha o la carrera (Minetti et al., 2012; Pavei et al., 2015) (Figura 4). Durante la carrera, el $C$ es independiente de la velocidad (Pavei y Minetti, 2016) y en terreno plano a velocidad constante es del orden de $4 \mathrm{~J} \mathrm{~kg}^{-1} \mathrm{~m}^{-1}$ (Di Prampero, Atchou, Brückner, y Moia, 1986; Lacour y Bourdin, 2015; Margaria, 1938; Pugliese et al., 2018) (figura 4). Existen investigaciones que estiman que el $C$ se podría obtener en base a ecuaciones que consideren la velocidad, la aceleración y la pendiente (McNeill Alexander, 2002; Minetti, Ardigò, et al., 1994; Minetti y Pavei, $\underline{2018})$.

\section{PATRONES Y MODELOS MECÁNICOS}

Si restamos los gastos "de manutención" del cuerpo, las actividades musculares, y por ende la locomoción, son las que más gastan energía metabólica. Hay entonces una fuerte presión selectiva hacia la evolución de mecanismos de optimización de los patrones de movimiento. En esta sección mostramos los modelos mecánicos y los mecanismos minimizadores del gasto energético de los patrones más comunes de locomoción.

\section{Marcha}

La marcha se representa por un modelo de péndulo invertido donde las extremidades inferiores son la estructura lineal y el $b_{\text {com }}$ es la masa unida a esta estructura (ver figura 5 ) (Margaria, 1976; Saibene y Minetti, 2003). Durante la primera fase del paso, desde el apoyo del talón y hasta cuando la pierna de apoyo se encuentra vertical, el bcom sube, aumenta su $E_{P} y$ disminuye su $E_{K}$. Durante la segunda fase, al revés, ocurre una disminución de la $E_{P}$ y aumento de la $E_{K}$ (Cavagna, Thys y Zamboni, 1976). 

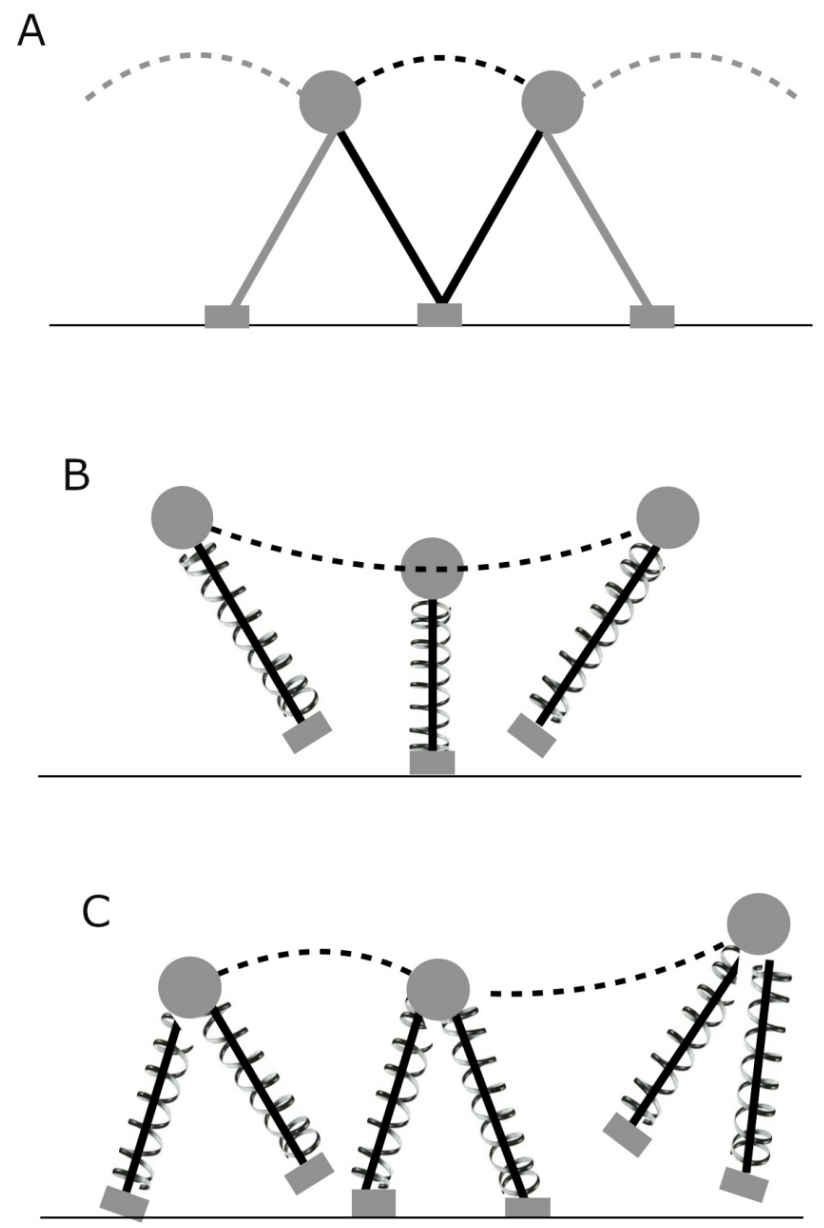

Figura 5. Modelos mecánicos de marcha (A: péndulo invertido), carrera y trote (B: masa-resorte), skipping y galope (C: péndulo y resorte). El círculo gris representa el centro de masa, y la línea discontinua su trayectoria. Fuente: elaboración propia.

Durante la marcha, la $E_{P}$ es alta cuando el bcom está encima del punto de contacto del cuerpo con el suelo, pero a partir de ese momento $E_{P}$ comienza a disminuir y la $E_{K}$ aumenta gradualmente. Cuando el punto de contacto vuelve a estar en frente de bcom disminuye la $E_{K} y$ aumenta la $E_{P}$. Las $E_{K}$ y $E_{P}$ se comportan en oposición de fase, generando un intercambio entre ellas que permite reciclar energía mecánica y ahorrar energía metabólica (ver figura 6) (Cavagna et al., 1976; Cavagna, Willems, Legramandi y Heglund, 2002). El parámetro que mejor representa el modelo pendular es el porcentaje de recovery $(R \%)$ (recuperación o reconversión en su traducción), que cuantifica la capacidad de ahorrar energía mecánica a través de la reconversión entre las $E_{K}$ y $E_{P}$ (Cavagna, 2017c; Cavagna et al., 1976). 

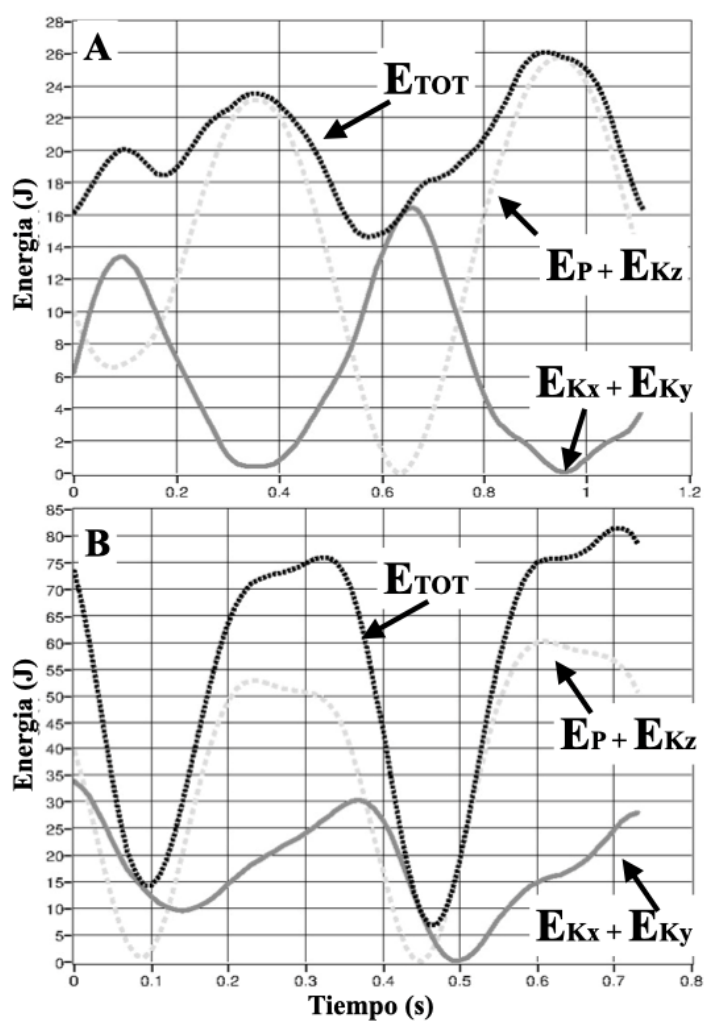

Figura 6. Ejemplo de trazados de energías del $\mathrm{b}_{\mathrm{com}}$ en un ciclo de marcha $(A)$ y de carrera (B). $E_{P}=$ Energía potencial gravitacional; $E_{K z}=$ Energía cinética (componente vertical); $E_{K x}, E_{K y}=$ Energía cinética (componentes horizontales); $\mathrm{E}_{\mathrm{TOT}}$ = Energía total. Fuente: elaboración propia.

En el modelo físico del péndulo ideal la reconversión entre las energías es total (100\%). En la marcha, el desface debido al movimiento pendular, puede llegar a un $R \%$ moderadamente elevado (hasta 60-70\%), siendo dependiente de la velocidad de marcha (Cavagna et al., 1976). El máximo $R \%$ se encuentra alrededor de la velocidad óptima de marcha (que normalmente coincide con la velocidad autoseleccionada), y va bajando a velocidades mayores o menores. El máximo $R \%$ coincide con el mínimo $C$ (Saibene y Minetti, 2003). Cuando es presentado en función de la velocidad de marcha, el $R \%$ presenta una característica de "U" invertida (ver figura 7). 


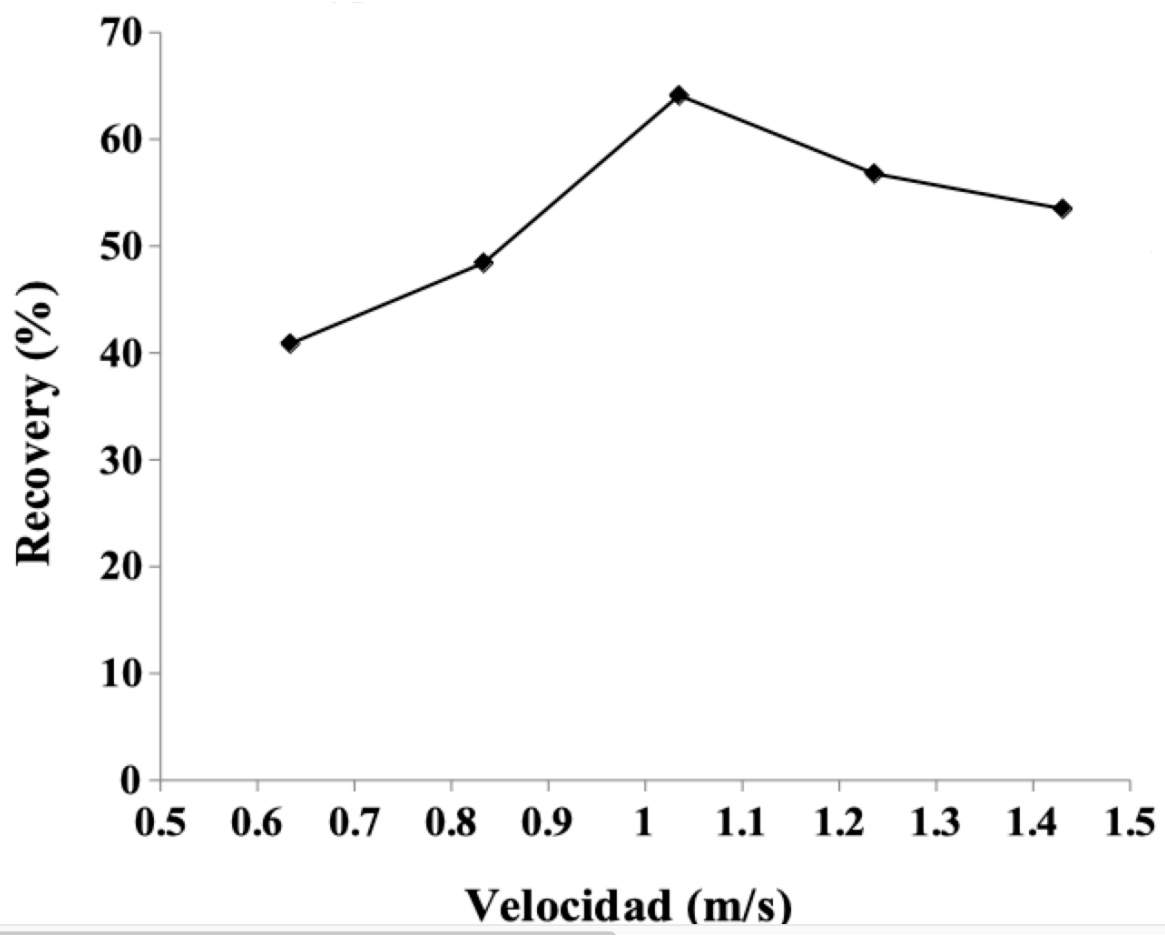

Figura 7. Porcentaje de recovery (recuperación) en función de la velocidad en un grupo de adultos, con su máximo a la velocidad de mínimo costo de transporte, alrededor de $1.1 \mathrm{~ms}^{-1}$ (Comparar con el $\mathrm{C}$ de la marcha presentado en la figura 3). Fuente: elaboración propia con base en (Bona et al., 2017).

Para describir el cálculo del $R \%$ (ecuación 8) se tiene que introducir dos nuevos parámetros: el trabajo vertical $W_{V}$ y el trabajo horizontal $W_{H}$. El $W_{V}$ es la suma de los incrementos de $E_{P}+$ la componente vertical de $E_{K}$. El $W_{H}$ es la suma de los incrementos de las componentes horizontales de $\mathrm{E}_{\mathrm{K}}$.

$$
R \%=W_{H}+W_{V}-W_{E X T} / W_{H}+W_{V}
$$

El $W_{E X T}$ depende de las variaciones de energía total $\left(E_{P}+E_{K}\right)$, que siempre son menores (o iguales) a la suma de las dos componentes (vertical y horizontal) tomadas por separado (figura 4).

Respecto a la contribución de la energía elástica $\left(E_{E)}\right)$ Ishikawa, Komi, Grey, Lepola y Bruggemann (2005) explican la contribución a partir de la posición de músculos durante las fases de la marcha. El comportamiento del gastrocnemio medial (estira durante la fase inicial de la fase de apoyo y luego permanece isométricamente durante la fase de apoyo final), el sóleo se alarga 
hasta el final de la fase de apoyo simple. Estos comportamientos sugieren que la $E_{E}$ se lleva a cabo no como un rebote (como ocurre en la carrera y saltos) en forma de resorte sino, como una acción de catapulta en el caminar. La interacción entre los fascículos musculares y los tejidos tendinosos desempeñan un papel importante en el proceso de liberación de energía elástica. Diferente de lo que observamos en la carrera, donde hay mayor participación de la $E_{E}$

\section{Carrera}

La locomoción humana presenta mecanismos no completamente esclarecidos y las teorías buscan explicarlos. En la carrera la teoría del sistema masa-resorte emerge como la más aplicable (figura 5) (Blickhan, 1989; McMahon y Cheng, 1990). El modelo masa-resorte es fundamentado en la parte de la física que estudia las condiciones de un oscilador armónico que trata sobre movimientos que se repiten en ciclos en torno de una posición de equilibrio. El comportamiento elástico de un resorte obedece a la Ley de Hooke (ecuación 9):

$$
F=K x
$$

Donde $F$ es la magnitud de la fuerza elástica producida, $K$ es la constante elástica del resorte (depende del tamaño y del material del resorte) $\left(\mathrm{N} \cdot \mathrm{m}^{-1}\right) ; x$ es la variación de longitud del resorte (deformación). Cuando un resorte es alargado o comprimido, una cantidad de energía potencial elástica $\left(E_{E}\right)$ puede ser almacenada y después liberada.

(Blickhan, 1989; McMahon y Cheng, 1990) fueron los primeros autores que propusieron el modelo físico-matemático para carrera y saltos: una masa conectada a la extremidad de un resorte para simular patrones mecánicos de saltos y carrera y comprender el mecanismo que minimiza la energía en estos movimientos. Ese modelo, masa-resorte, permite analizar la oscilación vertical del $b_{\text {com }}$. La oscilación del $b_{\text {com }}$ describe una trayectoria similar a aquella de un sistema elástico, siendo así denominado bouncing (rebote) (Cavagna, 2010). El sistema masaresorte representa el modelo biomecánico de la energía presente preponderantemente durante esa actividad, caracterizado por un sistema compuesto por un resorte capaz de almacenar energía elástica durante el contacto del pie con el suelo y liberarla durante el despegue (Blickhan, 1989).

Tal como se mencionó anteriormente las energías asociadas al $b_{C O M}$ son $E_{K}$ y $E_{P}$. A través de la observación del comportamiento de estas energías se hace posible identificar cómo la dinámica del $b_{\text {com }}$ minimiza el trabajo mecánico y maximiza la economía en la locomoción (Cavagna y Kaneko, 1977). Algunos parámetros del modelo masa-resorte son la rigidez vertical, 
frecuencia del sistema y asimetrías contacto-despegue, siendo este modelo probado y ampliamente analizado incluyendo diferentes situaciones y velocidades (Minetti, Ardigò, et al., 1994). A diferencia que, en la marcha, los patrones rebotantes (bouncing gaits) como la carrera la $E_{E}$ juega un papel importante en los mecanismos de ahorro de energía, su contribución se puede cuantificar de forma indirecta en humanos. Este hecho puede afectar el cálculo de la eficiencia mecánica de los patrones rebotantes (Minetti et al., 1999). En la carrera, la oscilación de la energía $E_{P}$ y $E_{K}$ es concomitantemente en fase (figura 4). O sea, no se observa una recuperación y conservación en el cambio entre las $E_{P}$ y $E_{K}$.

Es postulado que, durante la primera mitad de la fase de apoyo de la carrera, principalmente, músculos y tendones, se alargan almacenando energía elástica, en cuanto que los módulos de $E_{P}$ y $E_{K}$ disminuyen. Esa energía elástica almacenada como un resorte, es sumada, pero realmente es transformada en energía cinética potencial gravitacional, de modo gradual durante la segunda mitad de la fase de apoyo (Minetti, Ardigò, et al., 1994). Después de la fase de vuelo, parte de la energía total es nuevamente transformada en $E_{E}$ a través del alargamiento de la unidad músculo-tendón (Cavagna et al., 1964). Evidencias de $E_{E}$ almacenada y reconvertida también fueron encontradas en canguros y caballos (Alexander, 1989a). La característica anatómica del tendón permite ahorrar más o menos $E_{E}$, y a diferencia de los canguros, las ratas canguros ahorran menos $E_{E}$. Para obtener porcentajes de recuperación altos como los de los canguros, las ratas canguro necesitarían tendones extensores de tobillo mucho más delgados (Bienwener, Alexander y Heglund, 1981). El estudio en humanos es diferente al de animales, en estos últimos, a través de cirugía fue posible implantar electrodos para medir fuerza y electromiografía, lo que posibilitó una medida directa (Biewener, 1998). Los estudios en humanos, normalmente utilizan dinamometría, cinemática $2 \mathrm{D}$ y $3 \mathrm{D}$, electromiografía, ultrasonografía, fibras ópticas entre otros métodos (Alexander, 1984; Arndt, Komi, Brüggemann y Lukkariniemi, 1998; Fina et al., 2000; Ishikawa et al., 2005).

Los ligamentos del arco del pie también responden por el comportamiento elástico del sistema locomotor en la carrera. A pesar de la difícil estimación, evidencias indican que la $E_{E}$ es almacenada y liberada de los tejidos conectivos y contráctiles durante la carrera (Cavagna, 2010). Ker, Bennett, Bibby, Kester y Alexander (1987) muestran que además de las estructuras elásticas (tendones y tejidos conectivos) que contribuyen para la participación de la energía elástica en la carrera, los arcos plantares participan de la liberación de energía elástica también. Schleip y Müller (2013) estiman que para una persona de $70 \mathrm{~kg}$ a velocidad de $4.5 \mathrm{~m} \mathrm{~s}^{-1}$, el tendón de Aquiles almacena cerca de $35 \mathrm{~J}$ y los arcos plantares cerca de $17 \mathrm{~J}$. Investigadores siguen buscando informaciones respecto a las contribuciones de los tejidos conectivos en cuanto al 
ahorro de $E_{E}$, como formas de mejora de la performance y cuidados preventivos para los tejidos conectivos y todo el sistema miofascial (Magnusson, Langberg y Kjaer, 2010; Shrier, 2004).

Como muestra la figura 4, a diferencia de otros patrones rebotantes, en la carrera no hay una velocidad de mínimo costo. Cuando el costo es evaluado en función de la distancia recorrida, el comportamiento es lineal (Saibene y Minetti, 2003), lo que parece haber favorecido la capacidad de correr largas distancias en la evolución humana, animados por cuestiones de sobrevivencia, fuga de depredadores, búsqueda de alimentos y un lugar seguro. Asociado a la capacidad del eficiente mecanismo de absorción y liberación de energía elástica con estructuras que permiten actuar como un resorte (modelo masa resorte) a diferencia de la marcha (poca contribución elástica, modelo pendular) (Bramble y Lieberman, 2004; Carrier et al., 1984; Di Prampero et al., 1986).

Aún no hay acuerdo sobre las determinantes mecánicas de la economía de la carrera en el hombre. Parámetros espacio-temporales, velocidad, aceleración de gravedad y planos inclinados han sido ampliamente estudiados (Cavagna, 2010; Pavei et al., 2015). Si solo se toman parámetros biomecánicos internos estos no consiguen explicar la economía de carrera (Kyrolainen, Belli y Komi, 2001). Específicamente la economía de carrera es definida como el consumo de oxígeno para una determinada velocidad submáxima de carrera, y puede ser responsable por hasta $30 \%$ del rendimiento de un atleta de media y larga distancia (Kyröläinen, Belli y Komi, 2001).

Así, para obtener un buen rendimiento en carreras de larga distancia, el atleta debe poseer alto consumo máximo de oxígeno ( $\mathrm{VO}_{2 \mathrm{MAx}}$ ), habilidad de mantenerse en un alto porcentaje de $\mathrm{VO}_{2 M A ́}$, y tener un pequeño gasto energético en determinada velocidad, siendo este último factor evaluado a partir de la economía de carrera. Por lo tanto, el atleta más económico será aquel capaz de mantenerse en una velocidad submáxima con el menor gasto de energía metabólica. Varios estudios demuestran que el atleta es más económico cuando está en una longitud y frecuencia de pisada cerca de la autoseleccionada (Cavagna, 2010).

Así el modelo masa-resorte es el mecanismo minimizador de energía de la carrera auxiliando en la comprensión e interrelación con los beneficios de la práctica de ese deporte. Esos beneficios han sido documentados en el campo de la salud, a través de la mejora en la capacidad cardiorrespiratoria, en la reducción de la presión arterial crónica, así como mejora del perfil lipídico. En cuanto al rendimiento deportivo, su entrenamiento implica mayor tolerancia a la fatiga, mayor consumo de oxígeno y mejora en la economía de movimiento (Capelli, Antonutto, Zamparo, Girardis y di Prampero, 1993; Costill, Branam, Eddy y Sparks, 1971). 


\section{Skipping (Galope Bípedo)}

Es un término paralelo que asemeja al galope de los animales. Ha sido descrita bioenergética y mecánicamente por primera vez en humanos por Minetti en el año 1998 (Minetti, 1998). Se presenta como una forma de andar observada con mayor frecuencia en niños (Roberton, 2013). El patrón de pasos se ejecuta con un paso y un salto en una pierna seguido por un paso y un salto en la pierna opuesta (similar a ir descendiendo por una pendiente muy inclinada). El patrón de skipping (galope) incorpora componentes de caminar y correr vista con una doble fase de apoyo, vuelo y apoyo de una sola extremidad, con un modelo mecánico que une elementos de la marcha y de la carrera (figura 5) (Saibene y Minetti, 2003).

Una diferencia fundamental entre galopar y correr radica en la composición del paso; mientras correr repite un solo paso idéntico alternando entre las extremidades izquierda y derecha, galopar tiene dos pasos consecutivos pero diferentes realizados por cada miembro. El primer paso entra en contacto con la superficie antes del despegue de la otra extremidad, con esto se crea una breve fase de doble apoyo, seguida de salto del mismo pie. El aumento de la cadencia (número de pasos por minuto) muestra una mayor frecuencia de zancadas al comparar galope versus correr en todas las velocidades y gradientes (Minetti et al., 2012). El aumento de la cadencia y la disminución de la longitud del paso se debería directamente a las diferentes fuerzas de reacción del suelo entre los pasos (Heiderscheit, Chumanov, Michalski, Wille y Ryan, 2011). Esta información es compatible con una disminución del pico vertical de fuerza de reacción que acompaña las longitudes de paso más pequeñas observadas en el estudio de McDonnell y colaboradores en el 2017 (McDonnell, Willson, Zwetsloot, Houmard y DeVita, 2017). Minetti et al. (2012) informaron un costo metabólico invariablemente mayor al comparar con la carrera y es por esto, que el galope podría ser un patrón de locomoción viable ya que posee cargas musculo esqueléticas más bajas con un costo energético más alto en comparación con correr (Minetti, 1998; Minetti et al., 2012).

En el contexto del deporte y salud el galope, en comparación con la carrera, tiene picos de fuerzas de contacto de la articulación tibio-femoral y patelofemoral sustancialmente más bajas (30\% y 98\% respectivamente según Mc Donnel et al., 2019), al igual que impulsos lineales por paso y por kilómetro (es decir, cargas acumulativas) más bajas cerca al $37 \%$ en la articulación patelofemoral, además de un costo metabólico un 30\% más alto (McDonnell et al., 2019). Esto permite planificar ejercicios con menor carga articular y mayor gasto calórico. Si las fuerzas de contacto o los impulsos de la articulación de rodilla son factores causales de lesión, la sustitución de alguna cantidad de carrera con igual duración de galope puede permitir a los corredores reducir su potencial de lesión en la rodilla. 


\section{CONCLUSIÓN}

La aplicabilidad de todos esos conocimientos sobre la biomecánica de la marcha, carrera y galope tiene aplicación en distintas áreas del movimiento como educación física, fisioterapia o kinesiología, medicina, biología, física, ingeniería, entre otras áreas afines. Esto puede ser debido al hecho que actualmente diversas personas buscan el ejercicio físico como la caminata, carrera, o galope bípedo con objetivos de mejor calidad de vida, ejercicio físico o rendimiento atlético (Capelli, et al., 1993; Costill, et al., 1971; Fries, et al, 1994; Shipway y Holloway, 2010)

\section{REFERENCIAS}

Aiello, L. C., y Wells, J. C. K. (2002). Energetics and the Evolution of the Genus HOMO. Annual Review of Anthropology, 31(1), 323-338. doi: https://doi.org/10.1146/annurev.anthro.31.040402.085403

Alexander, R. M. (1976). Estimates of speeds of Dinosaurs. Nature, 261, 129-130. doi: https://doi.org/10.1038/261129a0

Alexander, R. M. (1989a). Elastic Mechanisms in the Locomotion of Vertebrates. Netherlands Journal of Zoology, 40(1-2), 93-105. doi: https://doi.org/10.1163/156854289X00200

Alexander, R. M. (1989b). Optimization and gaits in the locomotion of vertebrates. Physiological Reviews, 69(4), 1199-1227. doi: https://doi.org/10.1152/physrev.1989.69.4.1199

Alexander, R. M. (2003). Principles of Animal Locomotion. Princeton: Princeton University Press. Alexander, R. M., y Jayes, A. S. (1983). A dynamic similarity hypothesis for the gaits of quadrupedal mammals. Journal of Zoology, 201(1), 135-152. doi: https://doi.org/10.1111/j.1469-7998.1983.tb04266.x

Alexander, R. M. (1984). Elastic energy stores in running vertebrates. American Zoologist, 24(1), 85-94. doi: https://doi.org/10.1093/icb/24.1.85

Alexander, R. M., Jayes, A. S., Maloiy, G. M. O., y Wathuta, E. M. (1981). Allometry of the leg muscles of mammals. Journal of Zoology, 194(4), 539-552. doi: https://doi.org/10.1111/j.14697998.1981.tb04600.x

Alkner, B. A., y Bring, D. K.-I. (2019). Muscle activation during gravity-independent resistance exercise compared to common exercises. Aerospace Medicine and Human Performance, 90(6), 506-512. doi: https://doi.org/10.3357/amhp.5097.2019 
Arndt, A. N., Komi, P. V., Brüggemann, G. P., y Lukkariniemi, J. (1998). Individual muscle contributions to the in vivo achilles tendon force. Clinical Biomechanics, 13(7), 532-541. doi: https://doi.org/10.1016/s0268-0033(98)00032-1

Biancardi, C. M., Fabrica, C. G., Polero, P., Loss, J. F., y Minetti, A. E. (2011). Biomechanics of octopedal locomotion: kinematic and kinetic analysis of the spider Grammostola mollicoma. Journal of Experimental Biology, 214(20), 3433-3442. doi: https://doi.org/10.1242/jeb.057471

Biewener, A. A. (1989). Scaling body support in mammals: Limb posture and muscle mechanics. Science, 245(4913), 45-48. doi: https://doi.org/10.1126/science.2740914

Biewener, A. A. (1998). Muscle function in vivo: a comparison of muscles used for elastic energy savings versus muscles used to generate mechanical power1. American Zoologist, 38(4), 703717. doi: $\underline{\text { https://doi.org/10.1093/icb/38.4.703 }}$

Biewener, A. A. (1990). Biomechanics of mammalian terrestrial locomotion. Science, 250(4984), 1097-1103. doi: https://doi.org/10.1126/science.2251499

Biewener, A. A., y Patek, S. N. (2018). Animal locomotion (2 ${ }^{\text {nd }}$ ed.). Estados Unidos: Oxford Scholarship. doi: https://doi.org/10.1093/oso/9780198743156.001.0001

Blickhan, R. (1989). The spring-mass model for running and hopping. Journal of Biomechanics, 22(11-12), 1217-1227. doi: https://doi.org/10.1016/0021-9290(89)90224-8

Bona, R. L., Bonezi, A., da Silva, P. F., Biancardi, C. M., de Souza Castro, F. A., y Clausel, N. O. (2016). Electromyography and economy of walking in chronic heart failure and heart transplant patients. European Journal of Preventive Cardiology. doi: https://doi.org/10.1177/2047487316683284

Bona, R. L., Bonezi, A., da Silva, P. F., Biancardi, C. M., de Souza Castro, F. A., y Clausel, N. O. (2017). Effect of walking speed in heart failure patients and heart transplant patients. Clinical Biomechanics, 42, 85-91. doi: https://doi.org/10.1016/i.clinbiomech.2017.01.008

Bona, R. L., Gomeñuka, N. A., Storniolo, J. L. L., Bonezi, A., y Biancardi, C. M. (2019). Selfselected walking speed in individuals with transfemoral amputation: recovery, economy and rehabilitation index. European Journal of Physiotherapy, 22(3), 133-140. doi: https://doi.org/10.1080/21679169.2018.1561941 
Bramble, D. M., y Lieberman, D. E. (2004). Endurance running and the evolution of Homo. Nature, 432, 345-352. doi: https://doi.org/10.1038/nature03052

Capelli, C., Antonutto, G., Zamparo, P., Girardis, M., y Prampero, P. E. (1993). Effects of prolonged cycle ergometer exercise on maximal muscle power and oxygen uptake in humans. European Journal of Applied Physiology and Occupational Physiology, 66(3), 189-195. doi: https://doi.org/10.1007/BF00235092

Carrier, D. R., Kapoor, A. K., Kimura, T., Nickels, M. K., Scott, E. C., So, J. K., y Trinkaus, E. (1984). The Energetic Paradox of Human Running and Hominid Evolution [and Comments and Reply]. Current Anthropology, 25(4), 483-495. doi: https://doi.org/10.1086/203165

Cavagna, G. A. (2010). Symmetry and asymmetry in bouncing gaits. Symmetry, 2(3), 1270-1321. doi: https://doi.org/10.3390/sym2031270

Cavagna, G. A. (2017a). External, Internal and Total Mechanical Work Done During Locomotion. In Physiological Aspects of Legged Terrestrial Locomotion (pp. 129-138). Springer, Cham. doi: https://doi.org/10.1007/978-3-319-49980-2 6

Cavagna, G. A. (2017b). Physiological Aspects of Legged Terrestrial Locomotion: The Motor and the Machine. Springer, Cham. doi: https://doi.org/10.1007/978-3-319-49980-2

Cavagna, G. A. (2017c). Work, Efficiency and Elastic Recovery. In Physiological Aspects of Legged Terrestrial Locomotion (pp. 223-274). Springer, Cham. doi: https://doi.org/10.1007/978-3-319-49980-2 10

Cavagna, G. A., y Kaneko, M. (1977). Mechanical work and efficiency in level walking and running. The Journal of Physiology, 268(2), 467-481. doi: https://doi.org/10.1113/jphysiol.1977.sp011866

Cavagna, G. A., Saibene, F., y Margaria, R. (1963). External work in walking. Journal of Applied Physiology, 18(1), 1-9. doi: https://doi.org/10.1152/jappl.1963.18.1.1

Cavagna, G. A., Saibene, F., y Margaria, R. (1964). Mechanical work in running. Journal of Applied Physiology, 19(2), 249-256. doi: https://doi.org/10.1152/jappl.1964.19.2.249 
Cavagna, G. A., Thys, H., y Zamboni, A. (1976). The sources of external work in level walking and running. The Journal of Physiology, 262(3), 639-657. doi: https://doi.org/10.1113/jphysiol.1976.sp011613

Cavagna, G. A., Willems, P. A., Legramandi, M. A., y Heglund, N. C. (2002). Pendular energy transduction within the step in human walking. Journal of Experimental Biology, 205(21), 34133422. Recuperado de https://pubmed.ncbi.nlm.nih.gov/12324550/

Costill, D. L., Branam, G., Eddy, D., y Sparks, K. (1971). Determinants of marathon running success. Internationale Zeitschrift Für Angewandte Physiologie Einschließlich Arbeitsphysiologie, 29(3), 249-254. doi: https://doi.org/10.1007/BF01100536

Detrembleur, C., Dierick, F., Stoquart, G., Chantraine, F., y Lejeune, T. (2003). Energy cost, mechanical work, and efficiency of hemiparetic walking. Gait and Posture, 18(2), 47-55. doi: https://doi.org/10.1016/S0966-6362(02)00193-5

Detrembleur, C., Vanmarsenille, J. M., De Cuyper, F., y Dierick, F. (2005). Relationship between energy cost, gait speed, vertical displacement of centre of body mass and efficiency of pendulum-like mechanism in unilateral amputee gait. Gait and Posture, 21(3), 333-340. https://doi.org/10.1016/j.gaitpost.2004.04.005

Di Prampero, P. E. (2015). La locomozione umana su terra, in acqua, in aria : fatti e teorie. Milano: Edi.Ermes.

Di Prampero, P. E., Atchou, G., Brückner, J. C., y Moia, C. (1986). The energetics of endurance running. European Journal of Applied Physiology and Occupational Physiology, 55(3), 259266. doi: $\underline{\text { https://doi.org/10.1007/BF02343797 }}$

Elftman, H. (1940). The work done by muscles in running. American Journal of Physiology-Legacy Content, 129(3), 672-684. doi: https://doi.org/10.1152/ajplegacy.1940.129.3.672

Fedak, M. A., y Seeherman, H. J. (1979). Reappraisal of energetics of locomotion shows identical cost in bipeds and quadrupeds including ostrich and horse. Nature, 282, 713-716. doi: https://doi.org/10.1038/282713a0

Fenn, W. O. (1941). MUSCLE. Annual Review of Physiology, 3(1), 209-232. Recuperado de https://www.annualreviews.org/loi/physiol 
Fries, J. F., Gurkirpal S., Morfeld, D., Hubert, H. B., Lane, N. E., y Brown, B.W. (1994). Running and the development of disability with age. Annals of Internal Medicine 121(7), 502-509. doi: https://doi.org/10.7326/0003-4819-121-7-199410010-00005

Heiderscheit, B. C., Chumanov, E. S., Michalski, M. P., Wille, C. M., y Ryan, M. B. (2011). Effects of step rate manipulation on joint mechanics during running. Medicine and Science in Sports and Exercise, 43(2), 296-302. doi: https://doi.org/10.1249/MSS.0b013e3181 ebedf4

Hoyt, D. F., Wickler, S. J., Dutto, D. J., Catterfeld, G. E., y Johnsen, D. (2006). What are the relations between mechanics, gait parameters, and energetics in terrestrial locomotion? Journal of Experimental Zoology, 305A(11), 912-922. doi: https://doi.org/10.1002/jez.a.335

Ishikawa, M., Komi, P. V., Grey, M. J., Lepola, V., y Bruggemann, G. P. (2005). Muscle-tendon interaction and elastic energy usage in human walking. Journal of applied physiology, 99(2), 603-608. doi: https://doi.org/10.1152/japplphysiol.00189.2005

Ker, R. F., Bennett, M. B., Bibby, S. R., Kester, R. C., y Alexander, R. M. (1987). The spring in the arch of the human foot. Nature, 325, 147-149. doi: https://doi.org/10.1038/325147a0

Kyröläinen, H., Belli, A., y Komi, P. V. (2001). Biomechanical factors affecting running economy. Medicine \& Science in Sports \& Exercise, 33(8), 1330-1337. doi: https://doi.org/10.1097/00005768-200108000-00014

Lacour, J. R., y Bourdin, M. (2015). Factors affecting the energy cost of level running at submaximal speed. European Journal of Applied Physiology, 115, 651-673. doi: https://doi.org/10.1007/s00421-015-3115-y

Leonard, W. R., y Robertson, M. L. (1997). Rethinking the energetics of bipedality. Current Anthropology, 38(2), 304-309. doi: https://doi.org/10.1086/204614

Magnusson, S. P., Langberg, H., y Kjaer, M. (2010). The pathogenesis of tendinopathy: balancing the response to loading. Nature Reviews Rheumatology, 6(5), 262-268. doi: https://doi.org/10.1038/nrrheum.2010.43

Margaria, R. (1938). Sulla fisiologia e specialmente sul consumo energetico della marcia e della corsa a varie velocita ed inclinazioni de1 terreno. Atti Accad. Naz. Lincei Mem. Classe Sci. Fis. Mat., 7, 299-368. 
Margaria, R. (1976). Biomechanics and energetics of muscular exercise. Oxford: Oxford University Press, USA.

Margaria, R., Cerretelli, P., Aghemo, P., y Sassi, G. (1963). Energy cost of running. Journal of Applied Physiology, 18(2), 367-370. doi: https://doi.org/10.1152/jappl.1963.18.2.367

McDonnell, J., Willson, J. D., Zwetsloot, K. A., Houmard, J., y DeVita, P. (2017). Gait biomechanics of skipping are substantially different than those of running. Journal of Biomechanics, 64(7), 180-185. doi: https://doi.org/10.1016/j.jbiomech.2017.09.039

McDonnell, J., Zwetsloot, K. A., Houmard, J., y DeVita, P. (2019). Skipping has lower knee joint contact forces and higher metabolic cost compared to running. Gait \& posture, 70, 414-419. https://doi.org/10.1016/i.gaitpost.2019.03.028

McMahon, T. A. (1984). Muscles, reflexes, and locomotion. Princeton University Press. Recuperado de http://books.google.com/books?id=1sz5501URh0C

McMahon, T. A., y Cheng, G. C. (1990). The mechanics of running: How does stiffness couple with speed? Journal of Biomechanics, 23(Suppl. 1), 65-78. doi: https://doi.org/10.1016/0021$\underline{9290(90) 90042-2}$

McNeill Alexander, R. (2002). Energetics and optimization of human walking and running: The 2000 Raymond Pearl memorial lecture. American Journal of Human Biology, 14(5), 641-648. doi: https://doi.org/10.1002/ajhb.10067

Mian, O. S., Thom, J. M., Ardigò, L. P., Narici, M. V., y Minetti, A. E. (2006). Metabolic cost, mechanical work, and efficiency during walking in young and older men. Acta Physiologica, 186(2), 127-139. doi: https://doi.org/10.1111/i.1748-1716.2006.01522.x

Minetti, A. E. (1998). The biomechanics of skipping gaits: A third locomotion paradigm?. Proceedings of the Royal Society B: Biological Sciences, 265(1402), 1227-1235. doi: https://doi.org/10.1098/rspb.1998.0424

Minetti, A. E. (2001a). Biomechanics: Walking on other planets. Nature, 409(6819), 467-469. doi: https://doi.org/10.1038/35054166 
Minetti, A. E. (2001b). Invariant aspects of human locomotion in different gravitational environments. Acta Astronautica, 49(3-10), 191-198. doi: https://doi.org/10.1016/S0094$\underline{\text { 5765(01)00098-4 }}$

Minetti, A. E., Ardigò, L. P., Reinach, E., y Saibene, F. (1999). The relationship between mechanical work and energy expenditure of locomotion in horses. Journal of Experimental Biology, 202(17). 2329-2338. Recuperado de https://pubmed.ncbi.nlm.nih.gov/10441084/

Minetti, A. E., Ardigò, L. P., y Saibene, F. (1994). The transition between walking and running in humans: metabolic and mechanical aspects at different gradients. Acta Physiologica Scandinavica, 150(3), 315-323. doi: https://doi.org/10.1111/j.1748-1716.1994.tb09692.x

Minetti, A. E., y Pavei, G. (2018). Update and extension of the "equivalent slope" of speedchanging level locomotion in humans: A computational model for shuttle running. Journal of Experimental Biology, 221(15). doi: https://doi.org/10.1242/jeb.182303

Minetti, A. E., Pavei, G., y Biancardi, C. M. (2012). The energetics and mechanics of level and gradient skipping: Preliminary results for a potential gait of choice in low gravity environments. Planetary and Space Science, 74(1), 142-145. doi: https://doi.org/10.1016/j.pss.2012.06.004

Minetti, A. E., Saibene, F., Ardigò, L. P., Atchou, G., Schena, F., y Ferretti, G. (1994). Pygmy locomotion. European Journal of Applied Physiology and Occupational Physiology, 68(4), 285290. doi: https://doi.org/10.1007/BF00571444

Pavei, G., Biancardi, C. M., y Minetti, A. E. (2015). Skipping vs. running as the bipedal gait of choice in hypogravity. Journal of Applied Physiology, 119(1). doi: https://doi.org/10.1152/japplphysiol.01021.2014

Pavei, G., y Minetti, A. E. (2016). Hopping locomotion at different gravity: metabolism and mechanics in humans. Journal of Applied Physiology, 120(10), 1223-1229. doi: https://doi.org/10.1152/japplphysiol.00839.2015

Pequera, G., Bona, R., Bonezi, A., Polero, P., y Biancardi, C. M., (2020), Cardiopulmonary excercise breath-by-breath data during locomotion in 6 healthy young subjects. Mendeley Data, 1. doi: http://dx.doi.org/10.17632/vmwrtj29kr.1 
Pontzer, H., Raichlen, D. A., y Sockol, M. D. (2009). The metabolic cost of walking in humans, chimpanzees, and early hominins. Journal of Human Evolution, 56(1), 43-54. doi: https://doi.org/10.1016/.j.jhevol.2008.09.001

Pugliese, L., Porcelli, S., Vezzoli, A., La Torre, A., Serpiello, F. R., Pavei, G., y Marzorati, M. (2018). Different Training Modalities Improve Energy Cost and Performance in Master Runners. Frontiers in Physiology, 9. doi: https://doi.org/10.3389/fphys.2018.00021

Ranisavljev, I., Ilic, V., Soldatovic, I., y Stefanovic, D. (2014). The relationship between allometry and preferred transition speed in human locomotion. Human movement science, 34, 196-204.

Roberton, M. A. (2013). Testing the validity of the Halverson developmental sequences for skipping. Research Quarterly for Exercise and Sport, 84(2), 198-205. doi: https://doi.org/10.1080/02701367.2013.784726

Roberts, T. J., Kram, R., Weyand, P. G., y Taylor, C. R. (1998). Energetics of bipedal running. I. Metabolic cost of generating force. Journal of Experimental Biology, 201(19), 2745-2751. Recuperado de https://pubmed.ncbi.nlm.nih.gov/9732329/

Rodman, P. S., y McHenry, H. M. (1980). Bioenergetics and the origin of hominid bipedalism. American Journal of Physical Anthropology, 52(1), 103-106. doi: https://doi.org/10.1002/ajpa.1330520113

Saibene, F., y Minetti, A. E. (2003). Biomechanical and physiological aspects of legged locomotion in humans. European Journal of Applied Physiology, 88, 297-316. doi: https://doi.org/10.1007/s00421-002-0654-9

Salisbury, T., Baptista, R., Fei, J., Susin, F., y Russomano, T. (2015). Physiological Aspects of Walking in Simulated Hypogravity. Journal of Exercise Physiology Online, 18(6),13-25. Recuperado de: http://repositorio.pucrs.br/dspace/bitstream/10923/12348/2/Physiological_Aspects_of_Walkin g_in_Simulated_Hypogravity.pdf 
Schleip, R., y Müller, D. G. (2013). Training principles for fascial connective tissues: scientific foundation and suggested practical applications. Journal of bodywork and movement therapies, 17(1), 103-115. doi: https://doi.org/10.1016/j.jbmt.2012.06.007

Schmidt-Nielsen, K. (1975). Scaling in biology: The consequences of size. Journal of Experimental Zoology, 194(1), 287-307. doi: https://doi.org/10.1002/jez.1401940120

Shipway, R., y Holloway, I. (2010). Running free: embracing a healthy lifestyle through distance running. Perspectives in Public Health, 130(6), 270-276. doi: https://doi.org/10.1177/1757913910379191

Shrier, I. (2004). Does stretching improve performance?: a systematic and critical review of the literature. Clinical Journal of sport medicine, 14(5), 267-273. doi: https://doi.org/10.1097/00042752-200409000-00004

Steudel, K. (1996). Limb morphology, bipedal gait, and the energetics of hominid locomotion. American Journal of Physical Anthropology, 99(2), 345-355. https://doi.org/10.1002/(SICI)1096-8644(199602)99:2<345::AID-AJPA9>3.0.CO;2-X

Steudel-Numbers, K. L. (2003). The energetic cost of locomotion: Humans and primates compared to generalized endotherms. Journal of Human Evolution, 44(2), 255-262. doi: https://doi.org/10.1016/S0047-2484(02)00209-9

Taylor, C. R. (1994). Relating Mechanics and Energetics during Exercise. Advances in Veterinary Science and Comparative Medicine, 38, 181-215. Recuperado de https://pubmed.ncbi.nlm.nih.gov/7801832/

Taylor, C. R., Heglund, N. C., y Maloiy, G. M. O. (1982). Energetics and mechanics of terrestrial locomotion. I. Metabolic energy consumption as a function of speed and body size in birds and mammals. Journal of Experimental Biology, 97, 1-21. Recuperado de https://pubmed.ncbi.nlm.nih.gov/7086334/

Taylor, C. R., Schmidt-nielsen, K., y Raab, J. L. (1970). Scaling of energetic cost of running to body size in mammals. American Journal of Physiology, 219(4) 1104-1107. Recuperado de https://journals.physiology.org/doi/pdf/10.1152/ajplegacy.1970.219.4.1104 
Willems, P. A., Cavagna, G. A., y Heglund, N. C. (1995). External, internal and total work in human locomotion. The Journal of Experimental Biology, 198(2), 379-393. Recuperado de https://pubmed.ncbi.nlm.nih.gov/7699313/

Wunder, B. A., y Morrison, P. R. (1974). Red squirrel metabolism during incline running. Comparative Biochemistry and Physiology Part A: Physiology, 48(1), 153-161. doi: https://doi.org/10.1016/0300-9629(74)90863-9 